\title{
Control of customer and supplier risks by the guardband method
}

\author{
J.-M. Pou ${ }^{1, \star}$ and L. Leblond ${ }^{2}$ \\ 1 DELTA MU, Centre d'affaires du Zénith - Le Trident E 48 rue de Sarliève, 63800 Cournon d'Auvergne, France \\ 2 PSA Peugeot Citroën, centre technique de vélizy A, 2 route de Gésy, 78943 Vélizy-Villacoublay Cedex, France
}

Received: 7 July 2014 / Accepted: 10 January 2015

\begin{abstract}
Beyond their evaluations, measurement uncertainties raise many questions about their use in the context of the declaration of conformity of "products and services". If different approaches have been developed over the past years, including the "capability approach", 2012 has seen the JCGM document \#106 being published. This paper just published, was taken as an international standard ISO/IEC Guide 98-4 by ISO in the very same year and has just been taken (2013) in the collection of French standards (NF ISO/IEC Guide 98-4). This approach is singularly different from traditional approaches in that it introduces Bayesian concepts in the world of Metrology that was hitherto relatively impermeable to it. With this new approach, metrologists discover that measure is not a science of discovery, but a science of confirmation (or denial) of an "a priori".
\end{abstract}

Keywords: JCGM 106, NF ISO IEC guide 98-4, Customer Risk, Supplier Risk, Guardband, capability, declaration of conformity, measurement uncertainty, bayesian approach

\section{Introduction}

The end of year 2012 has seen the new ISO/IEC Guide 98-4 standard "Part 4: the Role of Measurement Uncertainty in Conformity Assessment" being published. This standard is a logical sequel of nearly two decades of discussions around measurement uncertainties which are not, however, in general, the major concern of users. Indeed, it is not, by itself, a parameter which is directly related to them, except in the case of calibration laboratories that have to control in the best possible way the value of their calibration standards (and therefore the associated uncertainties) in order to perform their services. For "Mr. Everybody" it occurs because it casts doubt on the declaration of conformity. What really interests the passenger of an airplane or the patient being diagnosed, it is not the true value of the characteristic being measured (for a critical part of the aircraft engine or through an analysis of the concentration of tumor markers in the blood) but rather whether he stands (or not?) a realistic chance of landing or whether he needs care. In a world where measurements would be an exact representation of reality, this question of "chances" would not make sense. In this case, either the mechanical part or the analysis is "compliant" and user is serene, or it is "non-compliant" and he knows what to expect. Thanks to recent years, we realized that measures cannot be fair and that therefore reality cannot be reached... We may only find an interval determined by the measurement result and the uncertainty, wherein the desired value is expected to be, for a known confi-

\footnotetext{
^Correspondence: jmpou@deltamu.fr
}

dence level. This range of possible values for the desired characteristic can sometimes "go beyond" the interval of specified tolerances. This "excess" raises doubts as for the conclusion given on the basis of the result: "Compliant or Non-compliant ?" and the Metrologist must manage to quantify these doubts.

\section{Prior to ISO/IEC Guide 98-4 standard}

This issue does not obviously occur only today, with this new standard. The problem is known and has been the subject of various strategies applied today.

\subsection{ISO 14253-1 (1998) international standard, geometrical product specifications (GPS) - inspection by measurement of workpieces and measuring instruments - Part 1: Decision rules for providing conformance or non-conformance with specification}

The international normative referential to address the problem of considering uncertainty in the declaration of conformity is, since 1998 (renewed in 2013), the ISO 14253-1 standard. ISO 14253-1 standard relies generally on a strategy that could be called "Minimum Risk" (which does not mean "Zero Risk" that we will never be able to reach!). The block diagram (Fig. 1) for taking into account uncertainty has become a classic, without reaching agreement. Indeed, it turns out that subtracting uncertainty 


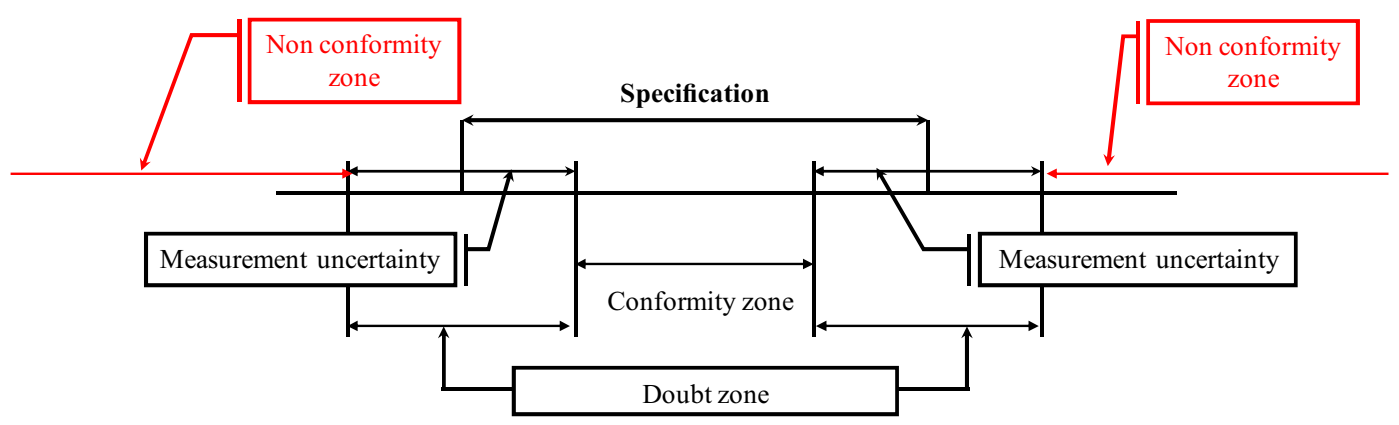

Fig. 1. Standard ISO 14253-1.

from specification often leads to almost impossible situations. Cases where the compliance area described by the standard is too small, or zero, are too common for manufacturers to effectively implement it... This is simply due to the fact that the specified requirements are rarely functional ones. Manufacturers know from experience that they can deviate (but by how much?) from specification without the function being called into question. It should be remembered that our "recipes" (specifications, guidelines, ... ) have often been established by experience. They initially rely on measured values (and not on real values) in tuning iterative processes. When a "recipe" has been tested, it is sufficient, in order to reproduce it, that measures stay in the instructions given for the function to be performed.

Would we turn now to true functional values, it is more than likely that the measurement uncertainty should "add" to the current tolerances rather than being subtracted from them. This would better respect the industrial reality even if advocated "sum" is not a real one, as we shall see below. It is this logic that the standards committee of the Union of Mechanics Standards followed in setting class boundaries (for "dimensional" instruments) since the release of ISO 14253-1. In order to apply it, it has been decided to add the calibration uncertainties of COFRAC accredited laboratories (substantially identical for each type of instrument) to the old class boundaries to define new ones...

But there is obviously a limit not to be exceeded, a functional limit to the true value. Thus, we cannot verify a given tolerance with any uncertainty. The latter must be adapted to the situation. From its 1994 version, ISO 9001 required that "the measurement uncertainties are known and consistent with the aptitude needed in the field of measurement". It is this concept of aptitude and/or compatibility of the measurement process with respect to the tolerance to check which is called "capability". This concept has just been the subject of an international standard, ISO 22514-7 (2012): "Statistical methods in process management - Capability and performance - Part 7: Capability of measurement processes".

\subsection{Capability}

What is at stake here is ensuring compliance with a contractual ratio between Tolerance and Uncertainty in order to conclude as to whether or not to use the measurement process to declare a product as complying.

Historically, the notion of capability, developed by car industry in the framework of Statistical Processes Control (SPC or MSP), concerns the production process. It comes in the context of mass-production for which it is impossible (and too expensive) to control $100 \%$ of the products and for which one rather seeks to control the rate of inexorably made "Non-Conforming" products. One then estimates "Customer Risk" i.e. the percentage of nonconforming delivered goods, which is known and accepted by the Customer. In order to achieve this goal, the capability of Processes features two major families of statistical parameters. The first one gives an indication of the adequacy between the natural dispersion of the Process and the tolerance (one generally refers to a coefficient named after $\mathrm{Cp}$ ) and the second one gives an indication of the eccentricity (Cpk in general). These two parameters $\mathrm{Cp}$ and $\mathrm{Cpk}$ are the ones that enable to determine the rate of "Non-Conforming" generated by a process under a normality assumption for example.

To be estimated, they need to make measurements which leads, of course, to uncertainties. In order not to have to worry about, one imposes negligible measurement uncertainties. A very severe ratio (10\%) between these measurement uncertainties and dispersion process is then imposed for the measurement process to determine $\mathrm{Cp}$ and Cpk parameters in a satisfactory way. This requirement understandable in this context drifted in one's mind towards a measurement process capability in general, which does not have much meaning. Indeed, the only ratio between Uncertainty and Tolerance cannot allow to know nor the "Customer Risk" or the "Supplier Risk", and the new NF ISO IEC Guide 98-4 finally clears this point at last.

But it is clear that this concept, apparently simple, has made its way into the minds and it is not uncommon today that a bound is enforced to this ratio in the context of calls for tenders for realizing the inspection of a set of measurement means. However, as the search for the Philosopher's Stone, each one looks in vain (again) for an optimal value to this bound.

Since this ratio does not meet, and will never meet alone, the legitimate question - What risks, "Customer" and "Supplier" measurement uncertainty generates? - it is often chosen by habit or by obligation, the laboratory 
being unable to do better. But what about the consequences of this chancy and often costly choice? We are going to see below how new NF ISO CEI Guide 98-4 standard may open perspectives that will allow for deciding in conscience the strategy to choose.

\subsection{The common practice}

The last available strategy, and probably the most often used, simply consists in ignoring uncertainties to declare conformity. This practice is so widespread (how doing otherwise some would say?) that, in France, COFRAC itself authorizes it, subject to an agreement between the client and the laboratory and under conditions of establishment of specification limits (see in particular the REF LAB 02, Rev7 document applicable from May, 1st, 2012, pp. 21-25, viewed and downloaded on website www.cofrac.fr). It defines the notion of "Declaration of Conformity with risks" in some of its documents and offers "typical" entries to indicate if they were or were not considered: "To declare the compliance or non-compliance, the interval of specifications has been reduced by the value of the uncertainty associated with the result" or "to declare the compliance or non-compliance, the uncertainty associated with the result was not taken into account". It will be noticed that by proposing to reduce the interval of specifications by the value of the uncertainty associated with the result, COFRAC simply invites the laboratory and its customer to comply with the recommendations of ISO 14253-1, as described above (Fig. 1).

COFRAC recognizes the sensitivity of this issue in an informative note at the end of paragraph 9.2.4.1. It introduced also the concepts of "Customer Risk" and "Supplier Risk" because, given the uncertainties, the interests of the one are in contradiction with the interests of the other. The "Customer Risk" is defined, in the world of metrology, as the risk to declare "Compliant" an object that would turn out to be in reality "Non-compliant". The "Supplier Risk" is in turn the risk to declare "Non-Compliant" an object that would be "Compliant". Without use of any mathematics up to now, we intuitively understand that decreasing one of the two risks increases the other one (and disproportionately!). It is therefore necessary to find a compromise between "Customer" and "Supplier" to determine a fair rule.

\subsection{A fundamental remark: the importance of words}

In industry, it is usual to enroll in a Customer-Supplier relationship and these words come easily to mind. Thus, the concepts of "Customer Risk" and "Supplier Risk" are directly and implicitly associated with one part or the other part of the contracting parties. This almost unconscious association led for instance COFRAC writing in his informative notes, "... according to the risk that the laboratory and its customers are willing to share". But what is really risk sharing?

In connection with any "metrological" service (Checking a measuring instrument) and in many cases related to tests, it's the applicant (the customer) that supports both risks actually... Regarding the consequences related to "Customer Risk", they could generate "non-compliance" in its production while using with good faith and trust, a supposedly compliant means that is not. But regarding the "Supplier Risk", the customer will be responsible for direct and indirect costs due to "non- compliance" which is not one! We can evoke the memory of astronomical costs in pharmaceutical industry, related to the management of apparently "non-compliant" means of measuring when it was most often the effect of "calibration uncertainty", the calibrated means being itself perfectly "compliant". In the context of this specific relationship, the risks are not shared, but they are the sole responsibility of the Customer. At the extreme, what is called intuitively "Provider Risk" may instead turn to a "business opportunity" for the laboratory by generating adjustments and recalibrations useless in reality.

\section{ISO/IEC Guide 98-4 standard: the optimal solution?}

Written by JCGM ${ }^{1}$, the JCGM 106 (2012) document became ISO/IEC Guide 98-4 the year of its release, and then French standard NF ISO IEC Guide 98-4 (2012). This demonstrates, if necessary, the weight of JCGM in the guidelines of international metrology. All those who have read the GUM (Document JCGM 100: 1995, corrected in 2008: Guide to the expression of uncertainty in measurement), written by the same committee, probably remembered a very technical and hardly approachable document. It is the same for this new document and it must be recognized that without a minimum knowledge and practice of Statistics, these highly technical documents are almost unreachable.

Beyond formulas and theorems (particularly Bayes who probably do not fail to mark the coming years) which this standard makes use of, it is easy to explain the basics of reflection that guide this standard and the conclusions it leads to. It would be damageable to close this document that may put off even the bravest, without having grasped its substance because it addresses the issue of compliance under a new and promising angle. Moreover, "Metrology" commission (X07B) in AFNOR, who voted enthusiastically for the endorsement of the new international standard based on this guide as a national standard, has now already gathered a group of experts to prepare a documentation booklet on the subject. The purpose of this booklet is to make concepts of this guide understandable and the first working meeting took place in the month of February 2013.

Remarkably, this standard has the merit of reconciling approaches presented above and will therefore slide quietly a current practice, maybe untreated or poorly mastered, towards a more rigorous and responsible approach.

\footnotetext{
1 http://www.bivi.metrologie.afnor. org/layout/set/popin/dictionnaire/ jcgm-joint-committee-for-guides-in-metrology.
} 


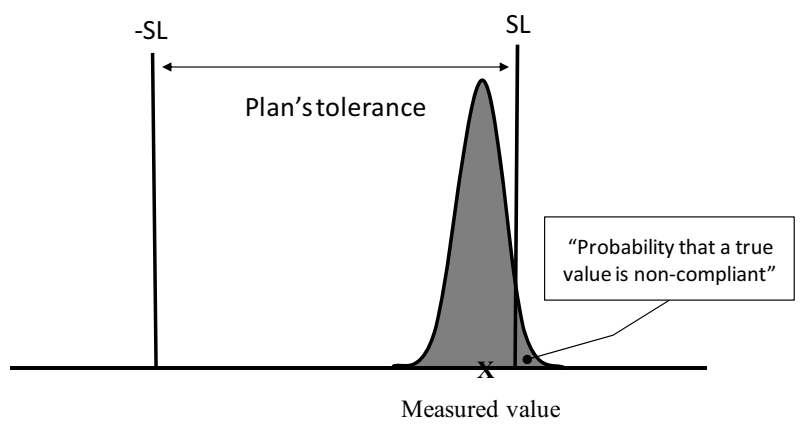

Fig. 2. Traditional view of "Customer Risk".

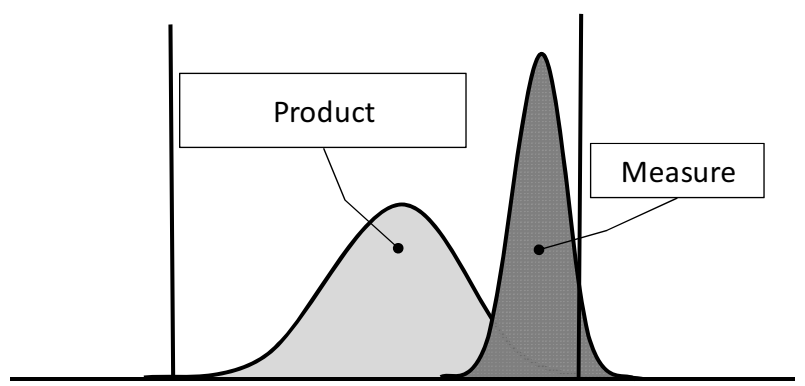

Fig. 3. Probability distributions associated to production process AND measurement process.

It relies on the concept of "Customer Risk" to define a strategy to hold, strategy consisting in defining, if necessary, a "guardband" that will guarantee the contractual "Customer Risk" despite unfulfilled capability. Take note however, to respect a proper form, that this approach is not really new for U.S. use it since long (see standard NCSL/ANSI Z540, evolution of MIL STD 45662A (1988)) and AFNOR evokes also these concepts in the specification FD X 07-022 - 2004 "Using measurement uncertainties: introducing some cases and customary practices".

\section{Definition of a “Customer Risk"}

It is now time to define "Customer Risk" as presented in the new standard. All too often, this concept of "risk" has been limited to determining the probability that a true value lies outside the limits of specification, based on a result of measurement and the associated uncertainty. This view is illustrated in Figure 2. But for the "Customer Risk" to exist, i.e. for a "non-compliant" product to be delivered, a Process must have produced it! The "Customer Risk" is therefore not only a measurement risk, but it was born from the combination of two random phenomena: the production of an item on one hand and the measurement error that appears during its control on the other hand. This vision, more in line with reality, has the merit of basing the reflection not only on measurement (the business of the metrologist) but also (and perhaps especially) on the Process. It is illustrated in Figure 3. Simply put, the "Customer Risk" is calculated as the product of the probability of achieving a "Non-compliant" product (Property of the production Process) by the (conditional) probability of a "Compliant" measure (that is within tolerance). This

$$
R C=\frac{1}{\sigma_{\text {Spic }} * \sigma_{\text {Mes }} * \Pi} * \int_{-\infty}^{-S L} \int_{-Z L}^{T L} e\left(\frac{-x^{2}}{2 * \sigma_{\text {Spéc }}^{2}}\right) * e\left(\frac{-(t-x)^{2}}{2 * \sigma_{\text {Mes }}^{2}}\right) d t d x
$$

Fig. 4. Determination of "Customer Risk" (where $x$ stands for true value and $t$, measured value).

product is expressed by a double integral, which makes quickly mathematical writing mysterious to common mortals (Fig. 4 shows the equation of "Customer Risk" for a Process according to a standard normal distribution). The "Supplier Risk" in turn is defined as the product of the (conditional) probability of measuring a "Compliant" product as "Non-compliant". It is subject to a similar calculation.

To get out of the mathematical difficulty as quickly as possible, and not to miss the essential, it is possible to consider things in a simpler almost playful angle: the numerical simulation.

\section{Simulation and risk determination}

If theory is essential to think about the phenomena and prove properties, numerical simulation allows to "materialize/visualize" phenomena at stake. We can, with the help of this technique, "see" things that reality deprives us to see. We will probably never know the real values of a Process, we will not know either the measurement error happening at each measure but one can quite use their respective properties to simulate possibilities of one and the other using a basic computer. It is then possible to replicate what goes on in reality and simply count the number of times the conjunction of the process and measurement are leading to take a wrong decision, in one direction (Customer Risk) or the other (Supplier Risk). Based on the number of carried simulations, we determine the rate of wrong decisions and these rates estimate the complicated formulas such as that in Figure 4. Then the cost of simplicity is unfortunately measured in computing time. One must indeed provide a huge number of simulations in order to obtain results with enough accuracy.

Note: Delta $M u$ has posted on its website (www. deltamu. fr) an Excel application to perform these simulations. The results were compared with values obtained by theoretical formulas.

\section{Risk and capability}

The concern that arises now is to move from the "capability" concept (Relationship between Tolerance and Uncertainty) to the "Customer Risk" concept. The idea relies on transforming "capability" (often contractual) into "Customer Risk" and considering that it is mainly the "Customer Risk" we need to guarantee, the capability of the measurement process being just an indicator without real meaning, as we have seen. Determining the "Customer Risk" associated with any coefficient of capability is done without difficulty when the properties of the Process are known. When they are not known, it is common to assume 


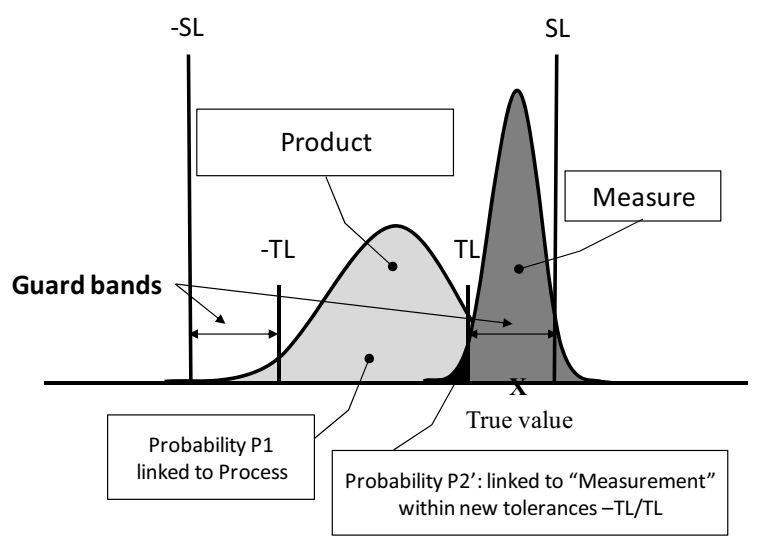

Fig. 5. "Guardbands" strategy.

them to be able to proceed to computations. Obviously, these approximations may have serious consequences (cf. Fig. 6).

When the capability of the process of measurement cannot be held, it is possible to compute "Guardbands" that reduce the initial tolerance and allow the "Customer Risk" initially accepted (Fig. 5) to be respected. But beware, this strategy can significantly increase the "Supplier Risk" and we have seen that the latter could also exist at the expense of the customer. Computer simulation allows again, by iterations, "Guardbands" and associated risks to be determined.

Note: The "Guardbands" notion rejoins the strategy proposed by ISO 14253-1. They are there directly defined by the measurement uncertainty and lead again to a "Customer Risk" which can be calculated as described above. If it is clear that this risk is low (apart from a totally degraded Manufacturing Process), the "Supplier Risk" is greatly increased. Figure 6 offers different determinations of "Customer" and "Supplier" Risks based on different assumptions about the Process and the measurement uncertainty. It may be noted that the assumptions on the Process impact significantly the value of each of the identified risks. One should therefore be very careful to these assumptions. .

\section{7 “Customer Risk”, “Supplier Risk”, "Guardband", how to choose?}

It is not always easy to decide on the risk it is possible to accept, as a customer, when you have to decide on the conformity of a measurement means or of a test. The "Customer Risk" within the meaning of the NF ISO IEC Guide 98-4, starts as soon as the error of the instrument exceeds the specification, commonly called Tolerated Maximum Error (TME) in the framework of metrological checks. But is the knowledge of this limit really consistent with the real need for functionality? In addition, when used, this TME is then theoretically "reduced" under a standard deviation form, often by a type B method, and introduced into a calculation of uncertainty. This TME to standard deviation transformation also suffers from approximations since the metrologist should consider a dis- tribution (without having to prove it!) in order to achieve it. A new doubt "weighs" then on the resulting standard deviation, probably due to ignorance of the actual distribution of error. To protect oneself, a uniform distribution is frequently used that aims at increasing standard deviation. One can easily compute that an error on the distribution, "Normal" instead of "Uniform" for example, leads to a gap on standard deviation of more than $40 \%$ ! Certainly, in this sense, $40 \%$ are majorating but who can guarantee that this error never occurs in the other direction (if the actual law is a "derived from arcsine law", error, minorating this time, is more than 20\%)? Therefore we accept this error today (or more!), and apparently without this causing a real problem.

The "Customer Risk" thus leads to overlook some real errors, but are they really significant when compared to other approximations made? Simulation allows us again to view the "real" errors of instruments that are not detected due to calibration uncertainties and that are involved in the "Customer Risk". We can then determine the maximum error (at a given confidence level) that may elude us. It is possible to determine the undetected maximum errors during checking. In the configuration of a Centered process (with a \pm 10 tolerance, without guardband with 6 standard deviations within the tolerance), there is a $5 \%$ probability that the unseen error exceeds 11.5 (instead of 10) and is less than -11.5 (instead of -10). One can legitimately ask if this 1.5 excess (11.5 to 10 ) beyond the specified limit is really significant by comparison with the knowledge of the real need for TME? In this case, the excess represents only $15 \%$ of the TME. But we have already seen that when using the TME by a type B method, we can make an error over $40 \%$, besides it is the variances that should be compared. It would have lead to a majorating $70 \%$ if the distribution is "Normal" instead of "Uniform", a a minorating $50 \%$ if it is "Derived from ArcSine". Under these conditions, the "Customer Risk" corresponding to $15 \%$ of the TME seems modest compared to these errors.

Note: It should be pointed out here that a "coarse" instrument error which would not have been detected during calibration/check (due to calibration uncertainty) can often be detected by user himself during monitoring operations. Thus, by implementing such operations (as requested by Quality referentials), Customer may "take a more important risk" in the context of metrological checks.

The "Supplier Risk", meanwhile, is and remains. It cannot be "fought" but at the price of repeated checks over the same means, thus increasing costs. Customer undergoes it "surreptitiously" and it generates unnecessary actions that cannot be easily identified. At a time when it becomes imperative to be efficient, these undue expenditures are probably not welcome...

So be careful not to slide too easily to proposals in NF ISO IEC Guide 98-4. The big risk is that providing laboratories apply the principle of the guardband, in good faith and intuitively thinking that the "Supplier Risk" is actually theirs. By imposing a capability coefficient, the customer is likely to impose herself/himself additional costs of fitting and re-verification means that do 


\begin{tabular}{|c|c|c|c|c|}
\hline \multicolumn{5}{|c|}{ Centered Process, Tolerance \pm 10} \\
\hline \multicolumn{2}{|c|}{$\begin{array}{c}\text { Number of standard deviations } \\
\text { "Process" in Tolerance }\end{array}$} & Strategy & $\begin{array}{c}\text { Customer } \\
\text { Risk }\end{array}$ & $\begin{array}{l}\text { Supplier } \\
\text { Risk }\end{array}$ \\
\hline \multirow{2}{*}{$\begin{array}{l}\text { Uncertainty }(U= \pm 2.5) \\
\quad(\text { Capability }=4)\end{array}$} & \multirow{2}{*}{4} & $\begin{array}{c}\text { Without } \\
\text { Guard band }\end{array}$ & $0.75 \%$ & $1.50 \%$ \\
\hline & & $\begin{array}{l}\text { With Guard band } \\
\qquad=U\end{array}$ & $0.03 \%$ & $9.96 \%$ \\
\hline \multirow{2}{*}{$\begin{array}{l}\text { Uncertainty }(U= \pm 5) \\
\quad(\text { Capability }=2)\end{array}$} & \multirow{2}{*}{4} & $\begin{array}{c}\text { Without } \\
\text { Guard band }\end{array}$ & $1.27 \%$ & $4.13 \%$ \\
\hline & & $\begin{array}{l}\text { With Guard band } \\
\qquad=U\end{array}$ & $0.03 \%$ & $32.69 \%$ \\
\hline \multirow{2}{*}{$\begin{array}{l}\text { Uncertainty }(U= \pm 2.5) \\
\quad(\text { Capability }=4)\end{array}$} & \multirow{2}{*}{6} & $\begin{array}{c}\text { Without } \\
\text { Guard band }\end{array}$ & $0.08 \%$ & $0.27 \%$ \\
\hline & & $\begin{array}{l}\text { With Guard band } \\
\qquad=U\end{array}$ & $\approx 0 \%$ & $3.20 \%$ \\
\hline \multirow{2}{*}{$\begin{array}{l}\text { Uncertainty }(U= \pm 5) \\
\quad(\text { Capability }=2)\end{array}$} & \multirow{2}{*}{6} & $\begin{array}{c}\text { Without } \\
\text { Guard band }\end{array}$ & $0.10 \%$ & $1.50 \%$ \\
\hline & & $\begin{array}{c}\text { With Guard band } \\
=U\end{array}$ & $\approx 0 \%$ & $22.72 \%$ \\
\hline
\end{tabular}

Risks are determined, in this table, by numerical simulation. They are therefore approximate values.

Fig. 6. "Customer" and "Supplier" Risks according to different configurations.

not deserve to be. There is a simple way out of this situation, simply search for a "Guardband" that leads to the best compromise between "Customer Risk" and "Supplier Risk". To do this, we should consider the weight of each risk in the organization of each customer and there is no golden rule.

\section{The solution: The minimization of the weighted sum of two risks}

The best compromise must take into account the economic impact (broadly defined) of the "realization" of these risks. What risk does the company support if she uses "non-compliant" means when she thinks it is "compliant"? What is the cost of a "No Compliance" management over concerned measurement means (impact studies, adjustments, recalibrations, immobilization, purchases, . . )? There is no point, of course, to compute euro cents near the actual costs incurred but weighing the two risks to have an objective view of the issue. We may define the "Customer Risk (cR)" has, either an impact of the same order as the "Supplier Risk (sR)" (Weights, denoted W, such as $\mathrm{WcR}=1$ and $\mathrm{WsR}=1$ ), or that it is five times larger $(\mathrm{WcR}=5-\mathrm{WsR}=1)$, or five times less severe $(\mathrm{WcR}=1-\mathrm{WsR}=5)$. From these weights, it is possible to define a "Guardband" that minimizes the weighted sum of two risks, regardless of any capability coefficient, but of course taking into account the measurement uncertainty. From a mathematical point of view, this minimization leads to the equation given in 10. Appendix Mathematical Development. But again, it is possible to solve this problem by numerical simulation.
Once the "Guardband" is determined, associated "Customer" and "Supplier Risks" are estimated and these two informations allow both objectively to compare the quality of the proposed service. It should also be clarified what is the maximum error that may not be seen in the case where the means is declared "Compliant", as seen above.

Indeed, a laboratory that would have significantly different uncertainty than another would determine the "Guardbands" that minimize the weighted sum (as his colleague), but the assessment of risks themselves in new tolerances (initial limits of specifications corrected by the "Guardband") would give different results that would allow to make a choice "in conscience". Figure 7 gives numerical values for virtual laboratories that would have different uncertainties for the same parameters of Process. Figure 8 shows the results that same laboratories would obtain if they simply determine the "Guardbands" to meet the "Customer Risk" induced by an arbitrary coefficient of capability ( $C=3$ in the example).

Note: for calculations of Figures 7 and 8, the risk weighting was set at $W c R=1$ and $\mathrm{WsR}=1$.

\section{Conclusion and prospect}

NG ISO IEC Guide 98-4 standard opens up therefore new horizons for metrology. Indeed, too focused on measurement, metrologist has perhaps not yet invested enough in the Process. However, to control its decisions, the new standard requires the metrologist to know the above Process since the "Customer Risk" and "Supplier Risk" depend very strongly on it. 


\begin{tabular}{lcccc}
\hline \multicolumn{4}{c}{ Centered Process, Tolerance \pm 10} \\
\hline $\begin{array}{l}\text { Number of standard deviations } \\
\text { "Process" in Tolerance }\end{array}$ & Strategy & $\begin{array}{c}\text { Customer } \\
\text { Risk }\end{array}$ & $\begin{array}{c}\text { Supplier } \\
\text { Risk }\end{array}$ \\
\hline Laboratory $1 / U= \pm 4$ & 4 & Minimization & $2.20 \%$ & $0.70 \%$ \\
Laboratory $2 / U= \pm 6$ & 4 & WcR + Ws $R$ & $2.95 \%$ & $0.87 \%$ \\
\hline Risks are determined, in this table, by numerical simulation. & They are \\
therefore approximate values.
\end{tabular}

Fig. 7. Example comparing risks obtained after minimizing the sum of weighted risks.

\begin{tabular}{|c|c|c|c|c|}
\hline \multicolumn{5}{|c|}{ Centered Process, Tolerance \pm 10} \\
\hline $\begin{array}{l}\text { Number of standard de } \\
\text { "Process" in Toler }\end{array}$ & $\begin{array}{l}\text { tions } \\
e^{-}\end{array}$ & $\begin{array}{c}\text { Strategy: } \\
\text { Customer Risk } \\
\text { preserved }\end{array}$ & $\begin{array}{l}\text { Customer } \\
\text { Risk }\end{array}$ & $\begin{array}{l}\text { Supplier } \\
\text { Risk }\end{array}$ \\
\hline Laboratory $1 / U= \pm 4$ & 4 & $C=3$ & $1.00 \%$ & $3.21 \%$ \\
\hline Laboratory $2 / U= \pm 6$ & 4 & $C=4$ & $1.00 \%$ & $7.90 \%$ \\
\hline
\end{tabular}

Fig. 8. Example comparing risks obtained after determining guardband preserving "Customer Risk" induced by Capability "C".

All these calculations are conditioned to an objective view of the Process. Changing an assumption about it is enough to substantially alter the conclusions and choices. After years of estimating measurement uncertainties, so we will have now to tackle the question of the properties of Processes to compute the risks. Nevertheless, it seems to us that this work can be relatively simple to achieve. Indeed, in the framework of metrological checks for instance, laboratories calibrate every day important amounts of instruments of the same type. It's the same for some testing laboratories carrying out "routine" tests on objects of the same kind. By analyzing the obtained experimental data, and taking into account the uncertainties (of calibration or of test) that came disturbing previous experimental data, it is possible to extract the distributions of desired characteristics. By sharing this information, it would be possible to provide to laboratories the data needed, on the Process side, to the assessment of risks. Here is a beautiful program of work for organizations such as COFRAC, AFNOR or the French College of Metrology, for example.

In addition, this paper deals with what NF ISO IEC Guide 98-4 calls "Global Risks", i.e. risks that are expressed when all the elements of a batch are checked. This is a rare situation in business but it is what happens under the management of a set of measuring instruments, especially for certified companies. The standard also provides guidance on "Specific Risks", i.e. the risk associated with the compliance of a part of a lot. For these specific risks, the philosophy is the same and is based on a Bayesian vision. This is to revise the measured value taking into account prior information on the Process to define a posterior distribution of probable values of the measured element. The risks are then calculated as proposed in Figure 2, but from the posterior distribution.

However, this standard does not address the statement of compliance from a sample taken from a lot and we regret it. Bayesian vision remains to be developed to address this situation very common in business.

\section{Appendix: Mathematical development}

random variable indicating the measurement results of a measurand on a physical characteristic (measurement process).

$Z$ : $\quad$ random variable indicating the "true" values of the measurand. $\nu, S$, and $T: \quad$ nominal, radius of specification and of tolerance.

Rate of non-compliant: $\pi=P(Z \notin[\nu-S, \nu+S])$

Customer risk: $\quad R_{C}(T)=P(U \in[\nu-T, \nu+T] \wedge$

$Z \notin[\nu-S, \nu+S])$

Supplier risk: $\quad R_{F}(T)=P(U \notin[\nu-T, \nu+T] \wedge$ $Z \in[\nu-S, \nu+S])$.

The probability density (resp. cumulative distribution) function of a standard normal distribution is denoted:

$$
\phi(x) \stackrel{\text { def }}{=} \frac{1}{\sqrt{2 \pi}} \exp \left(-\frac{1}{2} x^{2}\right)\left(\operatorname{resp} . \Phi(x) \stackrel{\text { def }}{=} \int_{-\infty}^{x} \phi(t) d t\right) .
$$

Main relationships used:

$$
\begin{aligned}
\forall(x, y, z) \in R^{3} \forall\left(\sigma_{Y / X}, \sigma_{X}\right) & \times R_{+}^{* 2} \Phi\left(\frac{y-x}{\sigma_{X}}\right) \\
& =\int_{-\infty}^{y} \frac{1}{\sigma_{X}} \phi\left(\frac{t-x}{\sigma_{X}}\right) d t, \\
\phi\left(\frac{y-z}{\sigma_{Y / X}}\right) \phi\left(\frac{z-x}{\sigma_{X}}\right)= & \phi\left(\frac{z-\left(\frac{y \sigma_{X}^{2}+x \sigma_{Y / X}^{2}}{\sigma_{X}^{2}+\sigma_{Y / X}^{2}}\right)}{\frac{\sigma_{Y / X} \sigma_{X}}{\sqrt{\sigma_{X}^{2}+\sigma_{Y / X}^{2}}}}\right) \\
& \times \phi\left(\frac{y-x}{\sqrt{\sigma_{X}^{2}+\sigma_{Y / X}^{2}}}\right) .
\end{aligned}
$$


The measurement process transforms the "true" value of the measurand linearly on average with a constant variability under normality assumption: $(U / Z) \sim \mathcal{N}(b Z+$ $\left.a, \sigma_{m}\right)$. The "true" values to be measured are normally distributed: $Z \sim \mathcal{N}\left(\mu, \sigma_{p}\right)$.

Therefore:

$$
\begin{aligned}
& U \sim \mathcal{N}\left(b_{1} \mu+b_{0}, \sqrt{b_{1}^{2} \sigma_{p}^{2}+\sigma_{m}^{2}}\right), \\
& Z / U \sim \mathcal{N}\left(\frac{b_{1}\left(U-b_{0}\right) \sigma_{p}^{2}+\mu \sigma_{m}^{2}}{b_{1}^{2} \sigma_{p}^{2}+\sigma_{m}^{2}}, \frac{\sigma_{m} \sigma_{p}}{\sqrt{b_{1}^{2} \sigma_{p}^{2}+\sigma_{m}^{2}}}\right), \\
& \pi=1-\Phi\left(\frac{\nu+S-\mu}{\sigma_{p}}\right)+\Phi\left(\frac{\nu-S-\mu}{\sigma_{p}}\right), \\
& P(U \notin[\nu-T, \nu+T])=1-\Phi\left(\frac{\nu+T-b_{1} \mu-b_{0}}{\sqrt{b_{1}^{2} \sigma_{p}^{2}+\sigma_{m}^{2}}}\right) \\
& +\Phi\left(\frac{\nu-T-b_{1} \mu-b_{0}}{\sqrt{b_{1}^{2} \sigma_{p}^{2}+\sigma_{m}^{2}}}\right) \\
& R_{C}(T)=\int_{r-[\nu-S, \nu+S]}\left[\Phi\left(\frac{\nu+T-b_{1} z-b_{0}}{\sigma_{m}}\right)\right. \\
& \left.-\Phi\left(\frac{\nu-T-b_{1} z-b_{0}}{\sigma_{m}}\right)\right] \frac{1}{\sigma_{p}} \phi\left(\frac{z-\mu}{\sigma_{p}}\right) d z \\
& R_{F}(T)=1-\pi-\int_{[\nu-S, \nu+S]}\left[\Phi\left(\frac{\nu+T-b_{1} z-b_{0}}{\sigma_{m}}\right)\right. \\
& \left.-\Phi\left(\frac{\nu-T-b_{1} z-b_{0}}{\sigma_{m}}\right)\right] \frac{1}{\sigma_{p}} \phi\left(\frac{z-\mu}{\sigma_{p}}\right) d z
\end{aligned}
$$

$\forall \lambda \in[0,1] \lambda R_{C}(T)+(1-\lambda) R_{F}(T)=R_{F}(T)$

$$
\begin{aligned}
& +\lambda\left[\Phi\left(\frac{\nu+T-b_{1} \mu-b_{0}}{\sqrt{b_{1}^{2} \sigma_{p}^{2}+\sigma_{m}^{2}}}\right)-\Phi\left(\frac{\nu-T-b_{1} \mu-b_{0}}{\sqrt{b_{1}^{2} \sigma_{p}^{2}+\sigma_{m}^{2}}}\right)\right] \\
& -\lambda(1-\pi) .
\end{aligned}
$$

The cancellation of the derivative of this expression with respect to $T$ provides the guardband minimizing the weighted sum of the two risks.

$$
\begin{aligned}
& \frac{\partial\left(\lambda R_{C}(T)+(1-\lambda) R_{F}(T)\right)}{\partial T}= \\
& \frac{\lambda}{\sqrt{b_{1}^{2} \sigma_{p}^{2}+\sigma_{m}^{2}}}\left[\phi\left(\frac{\nu+T-b_{1} \mu-b_{0}}{\sqrt{b_{1}^{2} \sigma_{p}^{2}+\sigma_{m}^{2}}}\right)\right. \\
& \left.+\phi\left(\frac{\nu-T-b_{1} \mu-b_{0}}{\sqrt{b_{1}^{2} \sigma_{p}^{2}+\sigma_{m}^{2}}}\right)\right] \\
& \quad \int_{[\nu-S, \nu+S]} \frac{1}{\sigma_{m}}\left[\phi\left(\frac{\nu+T-b_{1} z-b_{0}}{\sigma_{m}}\right)\right. \\
& \left.+\phi\left(\frac{\nu-T-b_{1} z-b_{0}}{\sigma_{m}}\right)\right] \frac{1}{\sigma_{p}} \phi\left(\frac{z-\mu}{\sigma_{p}}\right) d z .
\end{aligned}
$$

\section{References}

1. ISO 14253-1, 2013, Geometrical Product Specifications (GPS) - Inspection by measurement of workpieces and measuring equipment - Part 1: Decision rules for providing conformity or non-conformity with specifications

2. ISO 22514-7, 2012, Statistical methods in process management - Capability and performance - Part 7: Capability of measurement processes

3. ISO/IEC GUIDE 98-4, 2012, Uncertainty of measurementPart 4: Role of measurement uncertainty in conformity assessment 